\title{
Thermal Protection System to Enable Ice Giant Aerocapture Mission for Delivering both an Orbiter and an in situ Probe.
}

Principal Author: E.Venkatapathy, NASA, ethiraj.venkatapathy-1@nasa.gov, 650-248-1596.

Co-authros: D. Prabhu, AMA Inc., G. Allen, AMA Inc., and M. Gasch, NASA ARC.

Endorsing Co-signers: Michael J. Wright ${ }^{1}$, Paul F. Wercinski ${ }^{1}$, Michelle M. Munk ${ }^{2}$, Soumyo Dutta ${ }^{2}$, Steven L. Rickman ${ }^{3}$, Richard W. Powell ${ }^{4}$, Alexander Austin ${ }^{5}$, Adam Nelessen ${ }^{5}$, Marcus Lobia 5 . ( ${ }^{1}$ NASA ARC, ${ }^{2}$ NASA LaRC, ${ }^{3}$ NASA JSC, ${ }^{4}$ TEAMS3, ${ }^{5}$ JPL )

\section{Executive Summary and Recommendations}

Ice Giant Missions launched in the next decade can have significantly reduced trip times by incorporating aerocapture as the primary method for orbit insertion. Furthermore, a massefficient aerocapture system also enables the delivery of an orbiter, an atmospheric probe for in situ measurements to anchor global data collected by the orbiter, and possibly a lander at Triton. Aerocapture requires a Thermal Protection System (TPS) for the atmospheric entry, and it needs to be robust and mass efficient due to the size of the aeroshell required and the large heat load. Currently there are capable ablative thermal protection materials available, e.g., HEEET (Heatshield for Extreme Entry Environments Technology) and PICA (Phenolic-Impregnated Carbon Ablator) family. In this white paper, we show that the heat-shield TPS mass fraction can be as low as 5\% to as high as 20\% depending on total trip time reduction and other parameters. Further mass savings in the thermal protection system can be achieved with the use of conformal PICA, either standalone or in combination with HEEET with detailed design. We advocate the mission designers to consider aerocapture for Ice Giant missions so the science community can confidently consider missions to the Ice Giants to achieve unparalleled science in the coming decade.

1. Introduction: The use of chemical, or a combination of chemical and solar electric propulsion, for orbital insertion results in trip time estimates of 11 to 16 years to arrive at the Ice Giants [1]. However, there are limitations, especially for launch opportunities between 20292034, primarily due to the substantial amount of fuel required to slow down for any arrival

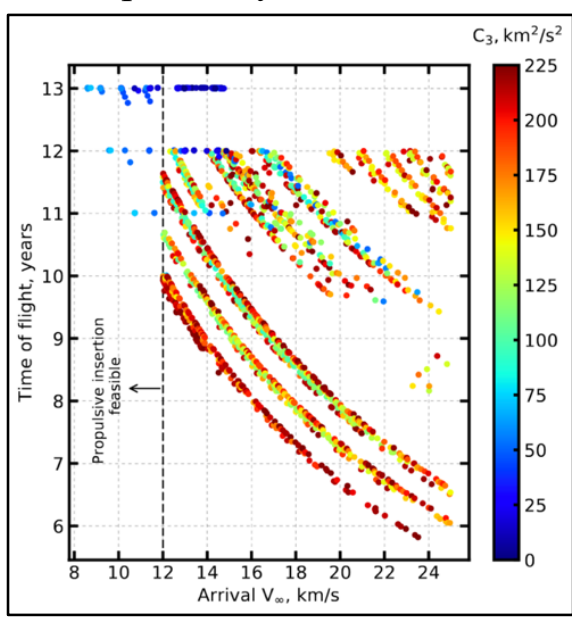

Fig. 1 Interplanetary trajectory velocity greater than $12 \mathrm{~km} / \mathrm{s}$, above which propulsive insertion is not possible [2]. Figure 1 (taken from the work of Girija et al. [2]) illustrates how the trip time could be reduced to roughly 8 years - a substantial reduction for a Neptune mission - with a moderate to low C3 launch opportunity and an arrival velocity of $22 \mathrm{~km} / \mathrm{s}$. An alternative is to use aerocapture for such arrival velocities. Ice Giant missions using aerocapture, especially Neptune missions to explore both Triton and Neptune, have been studied in the past [3]. The most comprehensive study of Uranus and Neptune aerocapture was based on technology available in 2005, and a slender body configuration, with a moderate $\mathrm{L} / \mathrm{D}(\sim 0.80)$ in order to achieve desired target apoapsis.

space to Neptune in 2030s-2040s. [2]. 
The penalty to protect the spacecraft during aerocapture is primarily the aeroshell mass (including the TPS mass which is the majority of the aeroshell mass). If the TPS mass can be substantially reduced, leading to a reduced mission mass, then shorter trip times with accommodation of larger science payload mass are possible, which in turn opens up the possibilities for very exciting Ice Giant missions. However, the use of a slender body aeroshell in the 2005 [3] study resulted in extreme entry conditions, and the challenges of finding and testing the appropriate TPS for the vehicle made such a concept both risky and expensive. The white paper by Dutta [4] provides more background on the feasibility of using aerocapture with a conventional blunt body with lower L/D for Ice Giant Missions. Advancements in thermal protection materials technology, guidance, navigation and control (GN\&C), and navigation technology, enable the use of existing rigid, lifting, blunt body configurations. Leveraging the work of $[2,5]$, and noting that

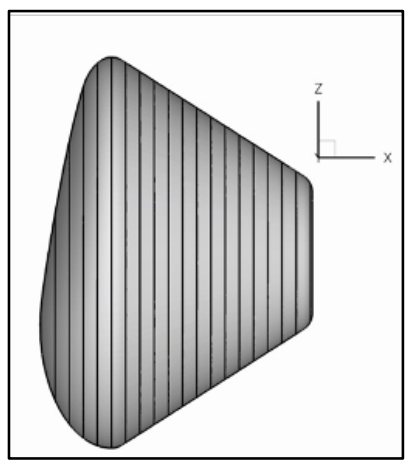

an $\mathrm{L} / \mathrm{D}$ of 0.4 is sufficient [3] to account for numerous uncertainties associated with the Ice Giant atmospheres, GN\&C, and aerodynamics, an asymmetric capsule vehicle (ACV, Fig. 2), based on the work of Brown et al. [5], was selected for the TPS evaluation. Instead of performing a point design, a broad design space with a range of entry velocities and ballistic coefficients was used in constructing several bounding aerocapture trajectories to demonstrate that currently available TPS materials are feasible and within testable limits. Sizing of the various TPS materials was then done on each of the bounding trajectories to evaluate the range of TPS mass required.

Fig.2. Asymmetric Capsule Vehicle(ACV) Geometry [5].

All lift-modulated aerocapture trajectories (roll and/or pitch/yaw control combination) can be bounded by 3DOF trajectories that have the lift vector either pointed towards the planet center (lift-down) or $180^{\circ}$ away from it (lift-up). Lift-down trajectories result in peak heat fluxes and pressures that are higher than the corresponding lift-up trajectories. Materials for the thermal protection system can then be selected for the lift-down trajectory with the highest heat flux. The materials selected can then be sized for the lift-up trajectory with the highest heat load. The present white paper, along with journal article [6] currently in preparation, addresses the TPS mass required to achieve reduction in trip time as low as $\sim 8$-years with arrival velocities of up to 22 $\mathrm{km} / \mathrm{s}$ at Neptune. The focus is primarily on the heatshield, which takes advantage of recent advances in two areas: 1) multidisciplinary optimization techniques used to design blunt body geometries for achieving the desired aerodynamic characteristics while minimizing the aerothermal environment to minimize TPS mass [5] ; and 2) recent advances in the development and maturation of a family of low-density carbon-phenolic TPS materials which have demonstrated performance capabilities to enable a robust and mass efficient aerocapture mission [7 - 11].

2. Aerocapture at Neptune to reach Triton: In the present study, a one-off CFD simulation is first performed for the asymmetric capsule shape (Fig. 2) using DPLR [12] to establish the aerodynamics parameters $-\mathrm{L} / \mathrm{D}=0.43, C_{D}=1.03$ and $C_{L}=0.44-$ for an angle of attack of $28^{\circ}$. Next, for these aerodynamic coefficients and vehicle attitude, 3DOF trajectory simulations are performed using TRAJ [13] for a $2200 \mathrm{~kg}$ entry mass of a $3 \mathrm{~m}, 4 \mathrm{~m}$ and $5 \mathrm{~m}$ diameter (all photographically scaled from the ACV aeroshell shown in Fig. 2. The resulting ballistic 
coefficients for the three different diameter aeroshells are $110 \mathrm{~kg} / \mathrm{m}^{2}, 172 \mathrm{~kg} / \mathrm{m}^{2}$, and $305 \mathrm{~kg} / \mathrm{m}^{2}$, respectively. 3DOF trajectories are computed for arrival velocities of $8 \mathrm{~km} / \mathrm{s}, 14 \mathrm{~km} / \mathrm{s}, 18 \mathrm{~km} / \mathrm{s}$, $22 \mathrm{~km} / \mathrm{s}$ (equivalently planet-relative entry velocities of $27 \mathrm{~km} / \mathrm{s}, 30 \mathrm{~km} / \mathrm{s}, 32 \mathrm{~km} / \mathrm{s}$ and $35 \mathrm{~km} / \mathrm{s}$ ). For each combination of ballistic coefficient and arrival velocity, two bounding trajectories are computed - for lift vector up and lift vector down. For arrival velocity of $22 \mathrm{~km} / \mathrm{s}$ the six bounding trajectories are shown in Fig. 3a. As mentioned before, these 3DOF trajectories bound all lift vector-modulated trajectories from 6DOF simulations. The main purpose of the selected entry parameters is to reduce the velocity through the atmospheric deceleration so that the resulting aerocaptured trajectory achieves a desired exit velocity of $25 \mathrm{~km} / \mathrm{s}$ relative to the planet. Figure $3 \mathrm{~b}$ shows the time histories of velocity for all 24 trajectories -3 ballistic coefficient $\mathrm{x} 4$ arrival velocities $\mathrm{x} 2$ lift states. It is clear that there is a successful velocity reduction independent of the design parameters and a delta $\mathrm{V}$ of up to $10 \mathrm{~km} / \mathrm{s}$ is possible with aerocapture to reach Triton.
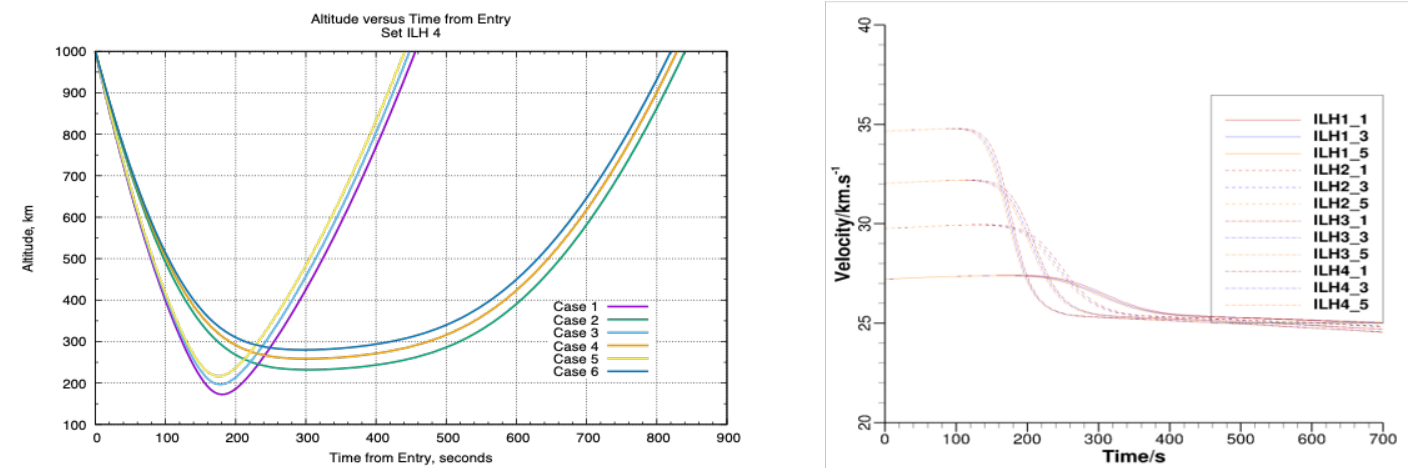

Fig. 3. (a) For an arrival velocity of $22 \mathrm{~km} / \mathrm{s}$, the aerocapture trajectory at Neptune to reach Triton for 3 different ballistic coefficients (or diameters of 3, 4 and $5 \mathrm{~m}$ ) with Lift-down and Liftup are shown. (b) All lift-down trajectories exit Neptune at $25 \mathrm{~km} / \mathrm{s}$ and a $10 \mathrm{~km} / \mathrm{s}$ delta Vis achieved through aerocapture.
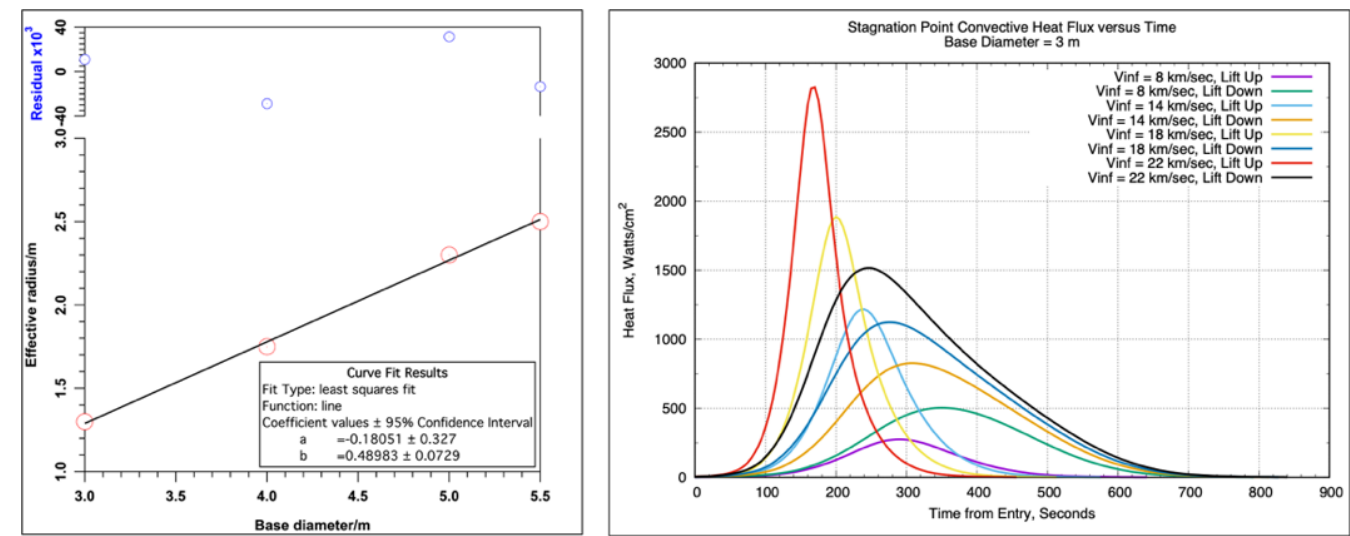

Fig. 4. (a) Radius of the equivalent hemisphere as a function of the base diameter of the flight configuration. The environments at the stagnation points of the two configurations are identical. (b) Stagnation point convective heating (hot-wall) time histories for the highest ballistic coefficient $\left(305 \mathrm{~kg} / \mathrm{m}^{2}\right)$, highest arrival velocity $(22 \mathrm{~km} / \mathrm{s})$, and two lift states - up and down. 
The next task is to determine the entry environments - pressure and heat flux - along each trajectory for TPS selection and sizing. For a symmetric blunt body at a zero angle of attack, correlations can be used to estimate the stagnation point heat flux, pressure and hence heat load once the trajectory is established. However, since the selected geometry is asymmetric and at an angle of attack, an engineering approach is adopted wherein the pressure and heat flux at the stagnation point of the asymmetric geometry are used to determine radius of an equivalent hemisphere. Further axisymmetric computations for the equivalent hemisphere are performed to verify that the pressure and heat flux at the stagnation points of the two geometries are the same. The relation between the equivalent or effective radius and the base diameter of the asymmetric 3D configuration are shown in Fig. 4a and the time histories of heat fluxes at the stagnation point of the equivalent hemisphere are shown in Fig. 4b. Having verified the equivalent hemispherical radius for all ballistic coefficients, the entry environments at the stagnation point of the hemisphere are determined along the entire trajectory.

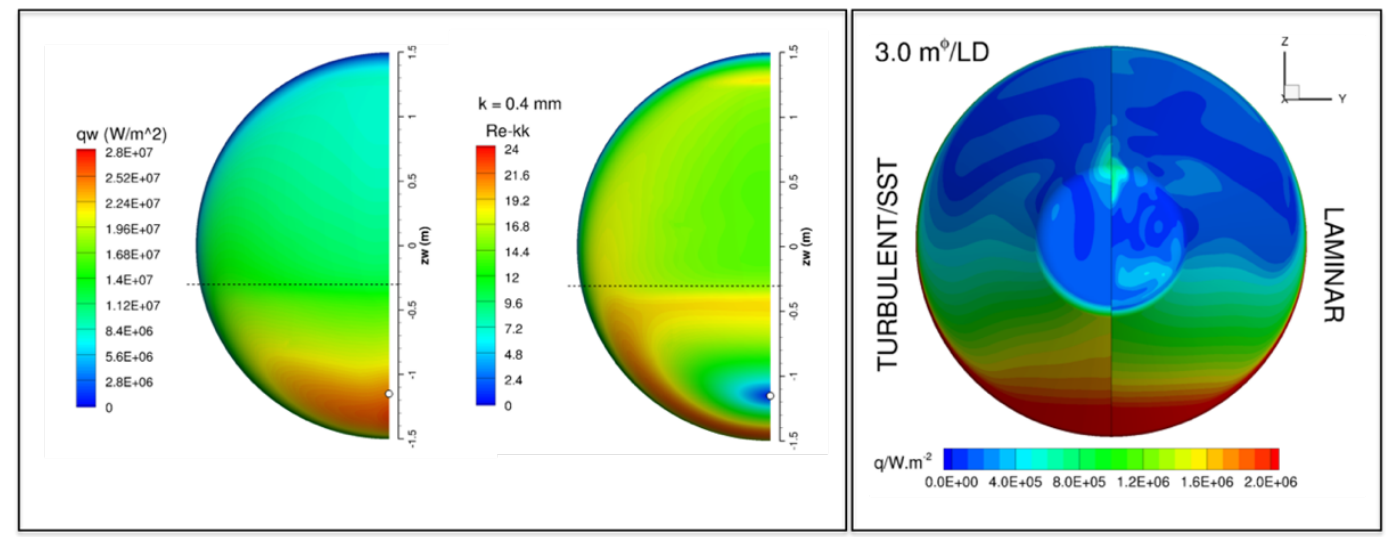

Fig. 5. Heat flux distribution shown on the left is for the most demanding of cases on an aerocapture trajectory at the lowest altitude. TPS material selection was based on the conditions shown. The center figure shows the $R e_{k k}$ distribution and the peak value is an order of magnitude lower than required for turbulent transition. In the figure on the right, the heat flux distribution on the backshell shown is indicative of the benign heating (between $20 \mathrm{~W} / \mathrm{cm}^{2}-200 \mathrm{~W} / \mathrm{cm}^{2}$ ).

Results of 3D CFD simulations for the asymmetric geometry at the peak heating points of lift down trajectories for the most demanding case of heat flux (highest ballistic coefficient (305 $\left.\mathrm{kg} / \mathrm{m}^{2}\right)$ and highest arrival velocity $(22 \mathrm{~km} / \mathrm{s})$ ) are shown in Figs. 5 and 6 . We note the following: (i) the acreage heating is well within the capability of ablative materials, with high heating confined to the stagnation region; (ii) even with a roughness height of 0.4 (typical of HEEET and PICA), the flow is unlikely to transition to turbulence; (iii) the backshell heating on the windward side is benign $\left(<200 \mathrm{~W} / \mathrm{cm}^{2}\right)$. From Fig. 6 we make the following observations: (i) the highest heating is around the windward shoulder and not at the stagnation point with the shoulder value being roughly $7 \%$ higher than the stagnation point value; (ii) since all of the trajectories are at a fixed L/D (angle of attack), the ratio of the heat flux between the stagnation point and any other point also remains nearly a constant ( this was verified with additional CFD runs). As a result, the heat flux and pressure as a function of trajectory at any point on the acreage can then be determined 

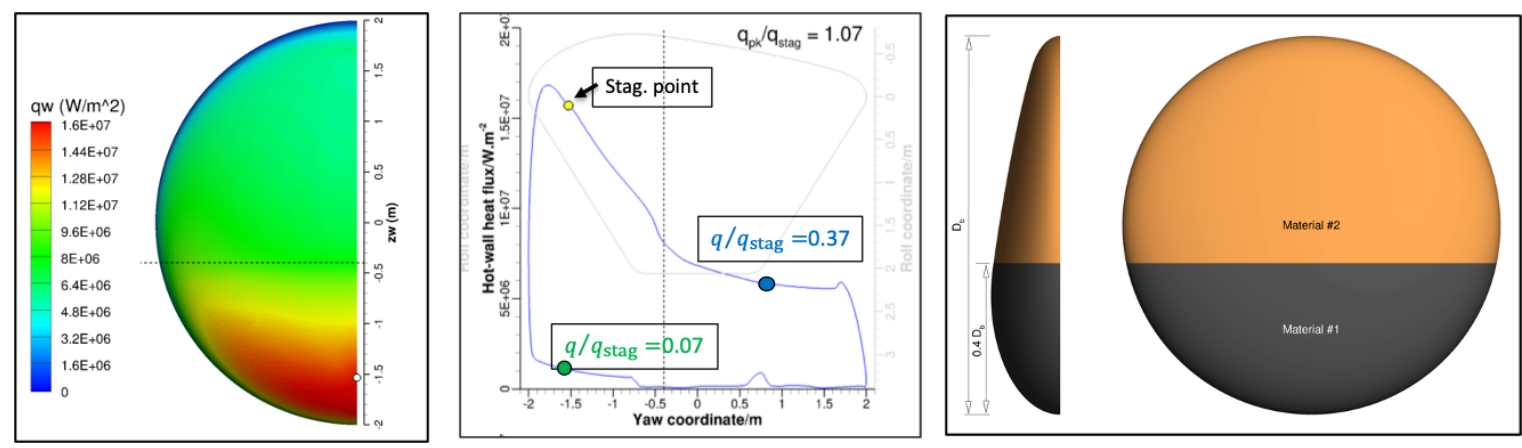

Fig. 6. Heat flux along the center symmetry line on heatshield and backshell surface of the ACV geometry, shown in the middle figure is extracted from the FD Computations (shown on the left) for the $4 \mathrm{~m}$ dia. at an arrival velocity of $22 \mathrm{~km} / \mathrm{s}$. Based on the pressure and heat flux distribution, instead of using a constant TPS thickness, a more mass efficient choice will be to use of two different TPS thickness or even two different TPS materials - one for the lower part (shown on the right) and another for the upper part.

3. TPS selection and Sizing: Based on the computed entry environment from all of the trajectories analyzed, TPS sizing was performed on three mature TPS materials suited for these environments. The first being the Heatshield for Extreme Entry Environment Technology (HEEET) TPS. HEEET is an advanced ablative TPS family constructed using 3D woven carbon and phenolic fibers. HEEET was developed to replace Carbon Phenolic, and it has been demonstrated in ground testing in extreme entry environments [7, 8]. A variant of HEEET is currently baselined as the heatshield for the Earth Entry Vehicle for the Mars Sample Return Campaign [7, 9]. Next is Phenolic Impregnated Carbon Ablator (PICA), which has substantial flight heritage on large aeroshells in a tiled configuration. It is qualified for heat fluxes less than $300 \mathrm{~W} / \mathrm{cm}^{2}$ in tiled configuration while as a single piece (without seams) it is capable of 1600 $\mathrm{W} / \mathrm{cm}^{2}$ heat flux and $1.5 \mathrm{~atm}$ pressure. In a tiled configuration PICA requires a seam - during technology development for the Crew Exploration Vehicle (the precursor to Orion/Artemis), an integrated PICA heatshield with a seam was explored. Its maturity was at TRL $\sim 5$, but this work was discontinued when the Avcoat ablative TPS was selected for Orion [10]. The third TPS considered is Conformal PICA (C-PICA), which is very similar in fabrication to rigid PICA but

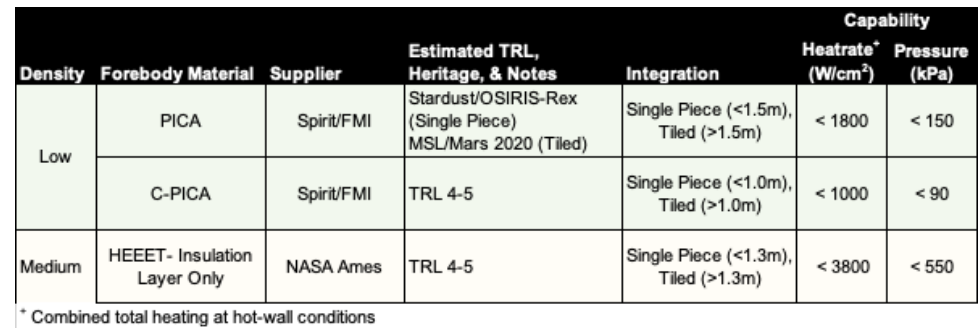

\section{Table 1. Ablative TPS materials and performance limit} more thermally efficient and mechanically complaint as the substrate uses carbon felt instead of rigid FiberForm. C-PICA was developed to be both mass efficient as well as compliant compared to rigid PICA, and it was matured to TRL 4+ [10-11].

Figure $7 \mathrm{a}$ shows the maximum heat flux at the windward shoulder of the ACVe configuration as a function of arrival velocity for the 3 ballistic coefficients considered. The heat fluxes are for trajectories with the lift vector down. The magnitude of the heat fluxes is such that HEEET can easily be used. However, HEEET might not be the most mass efficient material. Lower density materials in the PICA family could be used up to a certain limit on heat flux, say 1500 
$\mathrm{W} / \mathrm{cm}^{2}$. Imposing this limit in Fig. $7 \mathrm{a}$, it is clear that there are certain combinations of arrival velocity-ballistic coefficient coefficients for which a higher density material, such as HEEET, is necessary. However, the distribution of heat flux (Fig. 6) strongly suggests that a combination of HEEET and PICA or C-PICA would be a better option than seeking a single material solution. The thicknesses of the materials depend on the heat load. Figure $7 \mathrm{~b}$ shows the maximum heat load at the windward shoulder of the ACVe configuration as a function of arrival velocity for the 3 ballistic coefficients considered. The bounding heat loads is used when comparing different trajectories. The figure shows that the smaller the diameter, or equivalently, the larger the ballistic coefficient, the higher the heat load. These heat loads, in the range $\left(200,000 \mathrm{~J} / \mathrm{cm}^{2}-500,000\right.$ $\mathrm{J} / \mathrm{cm}^{2}$ ), are higher than those of typical EDL missions, such as Mars Entry or Sample Return missions, by couple of orders magnitude. To our knowledge, there has not been any TPS sizing performed for such demanding heat-load.

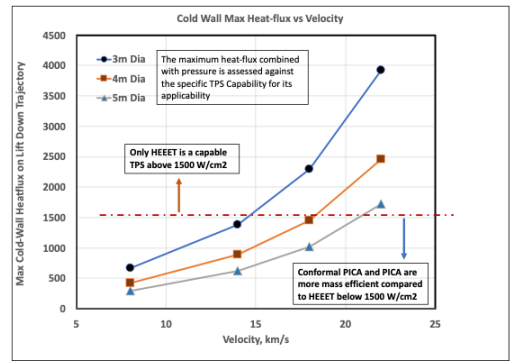

Fig. 7a. Max heat flux as a function of velocity.

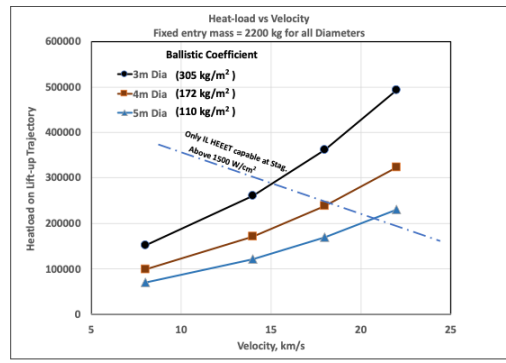

Fig. 7b. Total heat load as a function of velocity.

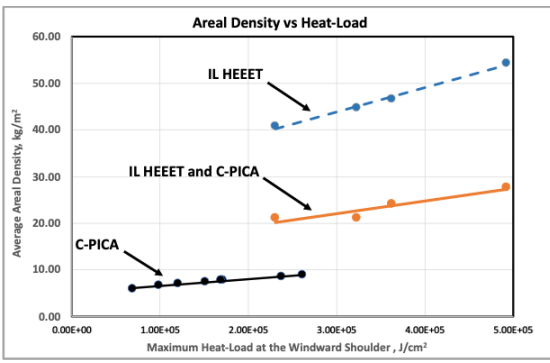

Fig. 7c. TPS areal density as a function of heat load

TPS sizing computations were performed using FIAT [15] for the HEEET and PICA family. Lift-vector-up trajectories were used in sizing the appropriate thickness for the chosen TPS materials. Summary of the results of the sizing computations using the three materials are summarized in Fig. 7c in terms of areal density. The results show that conformal PICA is the most efficient, when applicable, and has 4 to 5 times lower mass compared to HEEET. Since HEEET is substantially heavier, limiting its use where needed will allow for mass optimization. Even when conformal PICA is applicable for the entire heat-shield, a variable thickness sizing can achieve additional mass savings.

There are a number of simplifications and assumptions that were used in establishing the TPS mass and mass fractions, but the overall estimates have considerable conservatism and any refinement or point designs will result in lower mass for TPS than shown here. Firstly, all flight trajectories are based on 3DOF simulations and rely on engineering correlations for aerothermal environments. The heating environment as well as TPS sizing estimate use the same margin policy as missions do and hence there is conservatism to start with. The consideration of lift vector orientation - up and down - provides bounds for 6DOF trajectories. Secondly, a significant portion of the aerocapture trajectories will be in a transitional to free-molecular flow regime. Predicted aerothermal environments, either through CFD or engineering correlations, that are based on the assumption of a flow continuum will overestimate the heat flux and consequently, the total heat load. Lastly, the ballistic coefficient of $300 \mathrm{~kg} / \mathrm{m}^{2}$, considered to be the bounding cases, is roughly twice that of MSL/Mars 2020 (4.5 m diameter aeroshell); the ballistic coefficient of $172 \mathrm{~kg} / \mathrm{m} 2$ (4 $\mathrm{m}$ diameter ACVe aeroshell) is a more reasonable comparison. The conservative mass fractions computed using areal density from Fig. 7c., range between $(5 \%-20 \%)$. Even with the above conservatisms, the estimated mass fraction of the thermal protection system is very reasonable for 
heat loads that are an order of magnitude higher than typical large aeroshell EDL systems such as Artemis or Mars 2020.

4. Concluding Remarks and Recommendations: Our assessment of TPS for Ice Giant missions, using Neptune aerocapture as an example, illustrate that TPS materials currently available today are robust, mass efficient and capable of enabling Ice Giant aerocapture missions. The mass estimates across a broad range of conditions show TPS mass fractions ranging from 0.05 to 0.20 and can achieve much reduced trip time. The low TPS mass fraction we show for Aerocapture missions is very encouraging. We advocate the mission designers to consider aerocapture for Ice Giant missions so science mission planners can confidently consider aerocapture and design missions to the Ice Giants to deliver an Orbiter, along with a probe for Neptune and possibly a lander for Triton and obtain unprecedented information about the Neptune system to achieve unparalleled science in the coming decade. Since the PICA family of materials are relatively mature, their use as a heat-shield system requires only a small investment in technology maturation to complete the development from TRL (4-5) to TRL 6. Hence, we advocate for a technology maturation of conformal PICA to TRL 6 to be ready for the Ice Giant science mission planners to confidently evaluate and plan for an aerocapture mission in the coming decade.

\section{References:}

1. Elliot, J., et al. (2020), "Mission Design Prospects for the Ice Giants," Ice Giant Systems 2020, London.

2. Girija, A.P., et al. (2020), "Feasibility and Performance Analysis of Neptune Aerocapture using Heritage-Blunt Body Aeroshells" J Spacecraft and Rockets, 57(5), pp.1-18.

3. Lockwood, M.K., et al., "Aerocapture Systems Analysis for a Neptune Mission," NASA/TM2006-214300

4. Dutta, S., et al., "Aerocapture as an Enhancing Option for Ice Giants Missions," a White Paper Submitted for the Planetary Science Decadal Survey (2023 - 2032), July 2020.

5. Brown, J. L., et al., “An Asymmetric Capsule Vehicle Geometry Study for CEV,” AIAA 2007604, 45th AIAA Aerospace Sciences Meeting and Exhibit, Reno, Nevada, 2007. US Patent 7,431,242 B1: October 7, 2008.

6. Venkatapathy, E., Journal publication in preparation.

7. Stackpoole, M., et al. "Woven Thermal Protection System" (HEEET) US Patent 10,604,872: March 31, 2020.

8. Venkatapathy, E. "Mars Sample Return- Grand Challenge for EDL," ESI17 Technical Interchange Meeting: Thermostructural Modeling of TPS: NASA Ames Research Center, August 2018.

9. Blosser, M., Poteet, C. and Bouslog, S. "Space Vehicle Heat Shield Having Edgewise Strips of Ablative Material," (PICA on Edge) US Patent 9,051,063: June 9, 2015.

10. Gasch, M., et. al., "Development of Advanced Conformal Ablative TPS Fabricated from Rayon- and Pan-Based Carbon Felts," (2016) AIAA 2016-1414.

11. Stackpoole, M., et. al. "Method of Fabricating a Flexible, Low-Density TPS Material" (CPICA) US Patent 10,427,807: October 1, 2019.

12. Wright, M. J., White, T. R., and Mangini, N., NASA/TM-2009-215388, Oct. 2009.

13. Allen, G. A., Jr., et al. (2005) NASA/TM-2005-212847.

14. Venkatapathy, E., et al. (2020), Space Sciences Reviews, 216, 22.

15. Milos, F. S. and Chen, Y.-K. (2013) J Spacecraft and Rockets, 50(1), pp.137-149. 